\title{
Z Research S Suare \\ Spatiotemporal Heterogeneity Analysis of \\ Haemorrhagic Fever with Renal Syndrome in Anqiu City, China, 2000-2014
}

\section{Fuyan Shi}

Weifang Medical University

Changlan Yu

Anqiu City Center for Disease Control and Prevention

Fangyou Li

Anqiu City Center for Disease Control and Prevention

\section{Wenfeng Gao}

Affiliated Hospital of Weifang Medical College

\section{Liping Yang}

Xijing Hospital Affiliated Air Force Military Medical University

Suzhen Wang ( $\square$ wszdoc@126.com )

Weifang Medical University https://orcid.org/0000-0001-5926-1297

\section{Yongyong $\mathrm{Xu}$}

Air Force Military Medical University

\section{Research article}

Keywords: Spatiotemporal Analysis; Haemorrhagic Fever with Renal Syndrome; Meteorological Factors; Autoregressive Integrated Moving Average (ARIMA) Model; Prediction

Posted Date: May 23rd, 2019

DOI: https://doi.org/10.21203/rs.2.9754/v1

License: (c) (1) This work is licensed under a Creative Commons Attribution 4.0 International License. Read Full License 


\section{Abstract}

Background: The purpose of this study was to explore the dynamics of the occurrence of haemorrhagic fever with renal syndrome (HFRS) and find the potential spatiotemporal factors leading to the incidence of HFRS in Anqiu City. Methods: Monthly reported cases of HFRS and climatic data for 2000-2014 in Anqiu City were obtained. An autoregressive integrated moving average (ARIMA) model was used to fit the HFRS incidence prediction model and predict the epidemic trend in Anqiu City. Multiple linear regression method was used to analyze the temporal relationship between HFRS incidence and meteorological factors during the study period. Results: Spatial analysis results indicated that the annualized average incidence at the town level ranged from 2.18 to 6.09 per 100,000 among 14 towns, and the western towns in Anqiu City exhibited high endemic levels during the study periods. With high validity, the optimal ARIMA $(0,1,1) \times(0,1,1) 12$ model could be used to predict the HFRS incidence in Anqiu City in 2014. The monthly trend in HFRS incidence was negatively associated with temperature and precipitation and positively associated with average wind speed. Multiple linear regression models showed that precipitation and relative wind speed were key climatic factors contributing to the transmission of HFRS. Conclusions: This study provides evidence that the ARIMA model can be used to fit the fluctuations in HFRS frequency in Anqiu City. Our findings add to the knowledge of the role played by climate factors in HFRS transmission in Anqiu City and can assist local health authorities in the development/refinement of a better strategy to prevent HFRS transmission.

\section{Background}

Haemorrhagic fever with renal syndrome (HFRS) is an acute viral syndrome caused by infection with one variety of hantavirus. HFRS is an important infectious disease in developing countries [ 1,2]. In mainland China, HFRS remains a serious public health problem, with approximately 20,000-50,000 human cases reported annually, approximately $90 \%$ of the total cases worldwide[ 3,4$]$. Currently, HFRS is endemic in 28 of the 31 provinces in mainland China, and the highest incidence was reported in the middle and eastern part of China. Shandong Province, a highly developed coastal province, is one of the most severely affected provinces in China[ 5,6$]$. In order to effectively prevent the spread of HFRS, the Chinese Center for Disease Control and Prevention (CDC) established a surveillance system for HFRS in 2004 and created educational programmes for the general public. It is difficult to eliminate HFRS completely because many factors, such as diverse animal reservoirs and climate factors, may influence the control effects.

Many studies have suggested that epidemic modelling and forecasting can be important and effective tools to prevent and control HFRS[ 7-9]. The development and application of an HFRS incidence forecasting model are effective for improving the understanding of the epidemic characteristics of HFRS and could be helpful for the prevention and control of HFRS. Currently, statistical models used to predict the incidence of HFRS, including linear regression models[10,11], negative binomial multivariable regression[12], time series generalized additive models[ 13], generalized linear models (GLMs)[ 14], autoregressive integrated moving average (ARIMA) models[ 15, 16], generalized additive models (GAMs) [ 
17], nonlinear autoregressive neural networks (NARNNs) and ARIMA (ARIMA-NARNN) models[ 8], have been used to predict HFRS epidemics. Among those methods, the ARIMA model is the most common and useful method for modelling the temporal dependence structure of a time series. The ARIMA model can take into account changing trends, periodic changes, and random disturbances in a time series. In epidemiology, ARIMA models have been successfully used to predict the incidence of tuberculosis[ 18], dengue[ 19], as well as other infectious diseases [ 20].

Some studies predicted HFRS epidemics using ARIMA models and obtained a basis for targeted prevention and control measures[ 15,16$]$. These studies showed that ARIMA model had better predictive performance, but there was still some inconsistency between models and/or regions, which make researchers difficult to choose the appropriate one to predict HFRS epidemic. This inconsistency may be due to the fact that there are many influencing factors, such as immunization, temperature, humidity, elevation, and the local rat species [ $1,3,21,22]$. Therefore, the HFRS prediction model constructed from a particular region is not universal. To predict the epidemics of HFRS in a region accurately, a specific prediction model based on the actual data of the region need to be constructed.

Previous studies indicated that some areas in Shandong Province were moderate endemic areas with HFRS incidences between 5.0/100,000 and 30.0/100,000 from 1994 to 1998. However, few studies have been conducted to explore the dynamics of HFRS occurrence and determine the potential spatiotemporal factors leading to this disease in Anqiu City. In the present study, the spatiotemporal distribution patterns of HFRS cases were explored, the key climatic drivers of HFRS transmission were identified, and the optimal ARIMA model for predicting HFRS incidences was developed for Anqiu City. The results of this study can help predict the future trends of HFRS, which can be used to more accurately prevent and control HFRS in Anqiu City.

\section{Methods}

\section{Study area}

The study area covers Anqiu City in Shandong Province, which is located in the middle of the Shandong Peninsula. Anqiu City is located between latitudes $27^{\circ} 51^{\prime}$ and $28^{\circ} 40^{\prime}$ north and longitudes $111^{\circ} 53^{\prime}$ and $14^{\circ} 5^{\prime}$ east. Anqiu City is $217 \mathrm{~km}$ wide and $202 \mathrm{~km}$ long, with a total land area of $1760 \mathrm{~km}^{2}$. Anqiu City has a warm temperate continental climate influenced by the monsoon. The annual mean temperature in the study area is $12.2^{\circ} \mathrm{C}$, and the average annual rainfall is $646.3 \mathrm{~mm}$. Anqiu City consists of 14 towns with a total population of 0.95 million, among which the farmers account for 0.78 million. In Anqiu City, wheat, corn and peanut are the main crops, and most farmers reside less than $50 \mathrm{~m}$ from their farmlands.

Traditional farming methods provide an opportunity for wild rodent propagation, offering suitable living conditions and sufficient food resources to increase transmission of HFRS between rodents and from rodents to humans. 


\section{Data collection and management}

HFRS cases recorded monthly in Anqiu City from 2000 to 2014 were collected from the Anqiu City Center for Disease Control and Prevention (CDC). All HFRS cases were reported to the Anqiu City CDC through the National Infectious Disease Reporting System. All HFRS cases who were first diagnosed in hospitals or clinics were verified by Anqiu City CDC. The Anqiu City population data from 2000-2014 were determined based on information from the Anqiu City Bureau of Statistics. Envirnmental factors for Anqiu City during the study periods, including monthly average temperature, average air pressure, average sunshine, average wind speed, monthly precipitation, and average moisture, were collected from the Anqiu City Meteorological Bureau.

\section{Spatiotemporal analysis of HFRS case characteristics}

The spatiotemporal distribution characteristics, including the temporal and spatial distribution of HFRS cases from 2000 to 2014 in Anqiu City, were analysed according to the surveillance data from the infectious disease monitoring system. All HFRS cases were coded by administrative code using ArcGIS10.2 (ESRI Inc., Redlands, CA, USA), which were matched to the town-level polygon and point layers.

\section{Temporal trend analysis}

The ARIMA model is one of the most commonly applied time series prediction model. ARIMA was designed to address highly seasonal data. There was a strong seasonality trend in this study, and we constructed ARIMA models for monthly HFRS incidences in Anqiu City from 2000 to 2014. The ARIMA model was defined as the number of autoregressive lags $p$, moving-average lags $q$ and number of different passes $d$. The multiplicative seasonal ARIMA $(p, d, q) \times(P, D, Q) s$ model has apparent seasonal variation characteristics, which is an extended ARIMA model. Similar to ARIMA models, the seasonal parameters include seasonal autoregressive lags $P$, seasonal moving-average lags $Q$, seasonal differences D, and the length of the seasonal periods. The Augmented Dickey-Fuller Unit Root (ADF) test was applied to estimate the stationarity of the time series. If the time series is not stationary, an appropriate difference can be used to make the series stationary. The Box and Jenkins strategy was used to construct the seasonal ARIMA model in this study[23]. The ARIMA model analysis includes three main iterative steps: model identification, parameter estimation and the model evaluation. The autocorrelation functions (ACFs) and partial autocorrelation functions (PACFs) of the transformed data were utilized to determine the seasonal and non-seasonal orders and identify an appropriate ARIMA model. The conditional least squares method was applied to estimate the model parameters. In model diagnosis, white- noise- test methods were employed to check whether the residuals were independent and normally distributed. Several models may be constructed, and the selection of an optimal ARIMA model is necessary, which is usually based on the Akaike Information Criterion (AIC), normalized Bayesian Information Criterion (BIC) and Ljung-Box Q test. In addition, the mean absolute error (MAE), mean 
absolute percentage error (MAPE) and mean square error (MSE) were selected as the measures to evaluate the ARIMA model. The root mean square error (RMSE) was also used to access the accuracy of the models. All of these analyses were conducted using SPSS (version 17.0, SPSS, Chicago, IL, USA).

\section{Correlation analysis between meteorological factors and HFRS incidence}

Bivariate linear analysis and multiple linear analysis were used to model the relationship between meteorological factors and monthly HFRS incidence. The meteorological factors utilized in this study mainly included average temperature, monthly precipitation, average wind speed, and relative humidity.

\section{Results}

\section{Descriptive analysis of HFRS in Anqiu City}

708 cases were registered in Anqiu City during the 15-year study period. The highest average incidence rate was 15.05 per 100,000 people (in 2000), and the lowest average incidence rate was 1.15 per 100,000 people (in 2006), with an annual incidence rate of $69.20 / 100,000$. Among the total cases, $70.20 \%$ were male, and $29.80 \%$ were female, with a gender ratio (male vs. female) of 2.36. Among those patients, about $1.41 \%$ were children under 15 years old, $6.78 \%$ were old people over 64 years, and others were in 15-64 years group. Regarding occupation, $6.63 \%$ were workers (including forestry farmers and builders), $6.36 \%$ were students, and farmers accounted for $81.64 \%$.

The monthly HFRS cases is shown in Figure 1, which indicates that the occurrence of HFRS presented apparent seasonal character. There were two high peaks every year, the smaller epidemic occurred in spring between March and April and the larger one occurred in winter between October and November.

\section{Spatial distribution of HFRS incidence}

To account for the variations in incidence rates in small populations and areas, the annualized average incidence of HFRS per 100,000 in each town over the 15-year period was calculated. Furthermore, the annualized average incidences for each town were mapped in gradient colours. Figure 2(a-0) shows the annualized incidence for each town. Figure 2(a-o) shows that the annual incidence of HFRS gradually decreased from 2000 to 2006, and the lowest incidence of 1.15 cases per 100,000 occurred in 2006. Since 2007, the annual incidence of HFRS has increased gradually. Figure 2(p) shows the annualized average incidence for each town from 2000-2014. The annual average incidence at the town level ranged from 2.18 to 6.09 per 100 000, and among the 14 towns in Anqiu City, Huiqu, Wushan and Xing'an had high endemic levels with incidence rates between 5.28 and 6.09 per 100,000, while Shibuzi, Shidui, Zheshan and Linghe exhibited medium endemic levels, and other towns exhibited low endemic levels (Figure2p). 


\section{ARIMA model construction and evaluation}

\section{Model identification}

Monthly data of HFRS ranging from 2000 to 2013 in Anqiu City were used as the training set, and those for 2014 were used as the test data set. The sequence diagrams showed that the onset of HFRS has seasonal characteristics with a seasonal cycle every 12 months. As shown in Figure 1, the series showed that there was a non-stationary trend, so a 1st-order trend difference and a 1st-order seasonal difference were used to stabilize the average HFRS incidence. The converted series showed improved stability. Based on the distribution characteristics, we created eight models, $\operatorname{ARIMA}(0,1,0)(0,1,0){ }_{12}$, ARIMA $(0,1$, 1) $(0,1,1)_{12}$, ARIMA $(0,1,2)(0,1,2)_{12}$, ARIMA $(1,1,0)(1,1,0)_{12}$, ARIMA $(1,1,1)(1,1,1)_{12}$, ARIMA $(1,1,2)$ $(1,1,2)_{12}$, ARIMA $(2,1,0)(2,1,0)_{12}$, and ARIMA $(2,1,1)(2,1,1)_{12}$ (Table 1$)$.

\section{Parameter estimation}

According to the parameter estimation and goodness of fit test results (Table 1 and Table 2), we selected $\operatorname{ARIMA}(0,1,1)(0,1,1) 12$ model as the best one. The goodness of fit analysis indicated that there was no significant autocorrelation among residuals with different lags.

\section{Model testing and forecast analysis}

The monthly data in 2000-2013 was used to construct the ARIMA $(0,1,1)(0,1,1)_{12}$ model (Table 3 and Figure 3), and the monthly data in 2014 were used to test the model. The predicted data, actual data and the $95 \%$ confidence limit for the predicted data for 2014 are shown in Table 3 and Figure 3. The predicted data didn't exactly match the observed data, but the observed data fell within the $95 \%$ confidence interval of the predicted data.

\section{Multiple linear analysis of meteorological factors and monthly HFRS incidence}

Bivariate linear analysis results showed that monthly mean temperature, monthly precipitation and wind speed were related to HFRS incidence. A multiple linear analysis method was further used to model the relationship between monthly HFRS incidence and meteorological factors. The results showed that monthly precipitation, average wind speed and relative humidity were important factors in HFRS incidence in Anqiu City (Table 4).

\section{Discussion}


Epidemiological surveillance is one of the important interventions in the prevention and control of infectious diseases. Time series analysis methods are very useful for dynamic prediction and effective control of the diseases. Our study showed that the occurrence of HFRS presented apparent seasonality and that there were two annual peaks: the smaller peak occurred in spring between March and April, and the larger peak occurred in winter between October and November. From a public health perspective, our results support the need to carry out deratization campaigns in spring and winter around Anqiu City as well as enhance population immunity by vaccination throughout the year. In addition, we applied a multiplicative seasonal ARIMA $(0,1,1)(0,1,1)_{12}$ model to analyse the HFRS surveillance data in Anqiu City, China. Based on the results above, the $\operatorname{ARIMA}(0,1,1) \times(0,1,1)_{12}$ model is reliable with high prediction accuracy and can be used to predict the HFRS incidence in the subsequent year in Anqiu City. The prediction results suggested that the HFRS ARIMA model has strong ability to forecast and predict the incidence of HFRS in Anqiu City. Therefore, it is very important and necessary to learn about the knowledge of HFRS forecasts, which can help health agencies to allocate health resources reasonably.

Together with time series analyses, the application of GIS in our study provides a way to explicitly quantify HFRS and further determine the epidemiological characteristics accounting for the increasing disease risk. Our results showed that the potential risk areas were mainly concentrated around towns west of Anqiu City in recent years. The reason for this result may be that most towns in the west area are mountainous regions where the living environment is poor and villagers have weak health consciousness. Our study also indicated that an increase in HFRS incidence in Anqiu City was observed over the past 5 years, especially in 2012 when there was a small increase (Figure 2). This result may be due to the reconstruction and renovation of the old areas of Anqiu City in 2012, which included the renovation of a large number of houses, destroyed the rodent habitat, and made rats move frequently. These renovations also decreased the quality of the living environments of villagers and increased the chances of coming in contact with hantavirus, which further caused an increase in HFRS incidence. Based on our results, the government can allocate more health resources to high-risk areas and reduce the number of these resources used in low-risk areas to improve the effectiveness of interventions and the allocation of medical resources. Overall, both the temporal and spatial distribution patterns of HFRS in Anqiu City were studied. Disease prevention and control measures need to be strengthened in high-risk areas and during high-risk periods, which vary by month of the year.

In our study, correlation analysis and multiple linear analysis were used to further explore the relationship between environmental factors and HFRS incidence in Anqiu City. Our results showed that there was a negative association between temperature and HFRS incidence in Anqiu City, which was supported by other previous studies[24,25]. High temperatures may limit the time available to farmers for outdoor activity and work, thereby reducing the opportunity for contact between people and field mice, which is one of the most common agricultural pests and a natural vector of hantavirus. In addition, some studies have suggested that the breeding rate of rodents is highest at temperatures of $10-25^{\circ} \mathrm{C}$, which are favourable conditions for outdoor activity and work[25]. Anqiu City is located in a warm temperate continental monsoon climate zone, and the annual average temperature is $12.2^{\circ} \mathrm{C}$. However, inconsistent 
findings have been reported in other studies that indicated a positive association between temperature and the incidence of HFRS[24] [26]. This discrepancy might be due to different local conditions, such as different rodent compositions, different hantavirus serotypes, different environments and different climates in the study regions. Our data also indicated that precipitation was negatively associated with the incidence of HFRS; this finding is consistent with the findings of previous studies[27, 28]. Abundant precipitation could have a negative impact on rodents by destroying their habitats. In addition, frequent rainfall may decrease the likelihood of rodent-to-human contact, rodent-to-rodent contact, and virus transmission due to decreased rodent activity and reduced human exposure. However, several previous studies showed inconsistent findings of a positive association between precipitation and HFRS incidence[ 29-31]. There is no clear explanation for such differences, which may reflect the heterogeneity in local climate conditions. Further studies should be conducted in different regions to gain a better understanding of the impact of precipitation on HFRS. In addition, a positive association was obtained between average wind speed and HFRS incidence in our study, which was consistent with the results of a previous study[32].

The results of the multiple linear analysis showed that precipitation and wind speed were linearly related to the monthly numbers of HFRS cases and could be used to predict the number of HFRS cases in Anqiu City from 2000-2014. This study further provided practical evidence for the usefulness of correlation analysis and multiple linear regression analysis in HFRS prediction, with the optimum predictive climate variables being determined for different regions.

Some limitations of our study should be taken into account. First, we conducted a time series analysis without considering the factors that affected the occurrence of HFRS, such as animal reservoirs, human activities, farming patterns, and other socio-economic factors. Second, this study focused on only Shandong Province. Whether the model is suitable for other areas or infectious diseases needs further study.

\section{Conclusions}

In conclusion, HFRS incidence in Anqiu City demonstrated clear spatiotemporal heterogeneity from 20002014 and was primarily affected by meteorological elements and geographical factors. All of the above findings in our study support the use of spatiotemporal data to more accurately and effectively improve the understanding of the transmission patterns of HFRS. Our results can provide a quantitative basis to guide local control and prevention measures and have the potential to mitigate the risk and economic burden associated with HFRS. In addition, in order to more accurately predict the fluctuation of HFRS in Anqiu City in the next few years, it is necessary to construct a prediction model based on the data of continuous fluctuations in the region. Such prediction can help improve the the prevention and control of HFRS.

\section{List Of Abbreviations}


HFRS: Haemorrhagic fever with renal syndrome; CDC: Center for Disease Control and Prevention; GLM: Generalized linear model; ARIMA: Autoregressive integrated moving average; GAM: Generalized additive model; NARNN: Nonlinear autoregressive neural network; ADF: Augmented Dickey-Fuller; ACF:

Autocorrelation functions; PACF: Partial autocorrelation functions; AIC: Akaike Information Criterion; BIC: Bayesian Information Criterion; MAE: Mean absolute error; MAPE: Mean absolute percentage error; MSE: Mean square error; RMSE: Root mean square error.

\section{Declarations}

\section{Ethics approval and consent to participate}

Our study was approved by the Ethics Committee of Weifang Medical University and conformed to the provisions of the Declaration of Helsinki. Anyone who wants to access the raw data, please contact with Suzhen Wang.

\section{Consent for publication}

All authors have approved the manuscript for submission.

\section{Availability of data and material}

The datasets generated during and/or analysed during the current study are available from the corresponding author on reasonable request.

\section{Competing interests}

The authors declare that they have no competing interests.

\section{Funding}

This work was supported in part by the National Nature Science Foundation of China (No. 81872719, No. 81803337, No. 81573250), Shaanxi Key Industry Innovation Chain (2016KTZDSF02-07-01), Shandong Science and Technology Development Plan Project (2015 WS0067), and Weifang Medical University Doctor Start Fund (2017BSQD51).

\section{Authors' contributions}

S.Z. W and Y.Y. X conceived and designed the study. C.L. Y and F.Y. L provided the data. F.Y. S, L.P. Y and W.F. G analysed the data. F.Y. S wrote the first draft, implemented revisions and finalized the manuscript. 
F.Y. S, L.P. Y, W.F. G, C.L. Y and F.Y. L reviewed and edited the manuscript. All authors read and approved the final manuscript.

\section{Acknowledgements}

Sincere thanks to the local research teams from Anqiu City Center for Disease Control and Prevention (CDC) for their contribution to the survey. We are also indebted to the Anqiu City Bureau of Meteorology for providing climate data.

\section{References}

1. Jonsson CB, Figueiredo LTM, Vapalahti O: A Global Perspective on Hantavirus Ecology, Epidemiology, and Disease. Clinical Microbiology Reviews 2010, 23(2):412-441.

2. Vaheri A, Strandin T, Hepojoki J, Sironen T, Henttonen H, Mäkelä S, Mustonen J: Uncovering the mysteries of hantavirus infections. Nature Reviews Microbiology 2013, 11(8):539-550.

3. Lei Y, Fang LQ, Huang HG, Zhang LQ, Dan F, Zhao WJ, Zhang WY, Li XW, Cao WC: Landscape Elements and Hantaan Virus-related Hemorrhagic Fever with Renal Syndrome, People's Republic of China. Emerging Infectious Diseases 2007, 13(9):1301-1306.

4. Zhang S, Wang S, Yin W, Liang M, Li J, Zhang Q, Feng Z, Li D: Epidemic characteristics of hemorrhagic fever with renal syndrome in China, 2006-2012. Bmc Infectious Diseases 2014, 14(1):384.

5. Wang L, Wang T, Cui F, Zhai SY, Zhang L, Yang SX, Wang ZQ, Yu XJ: Hemorrhagic Fever with Renal Syndrome, Zibo City, China, 2006-2014. Emerging Infectious Diseases 2016, 22(2):274-276.

6. Wang T, Liu J, Zhou Y, Cui F, Huang Z, Wang L, Zhai S: Prevalence of hemorrhagic fever with renal syndrome in Yiyuan County, China, 2005-2014. Bmc Infectious Diseases 2015, 16(1):69.

7. Han SS, Kim S, Choi Y, Kim S, Kim YS: Air pollution and hemorrhagic fever with renal syndrome in South Korea: an ecological correlation study. Bmc Public Health 2013, 13(1):347-347.

8. Lin H, Zhang Z, Lu L, Li X, Liu Q: Meteorological factors are associated with hemorrhagic fever with renal syndrome in Jiaonan County, China, 2006-2011. International Journal of Biometeorology 2014, 58(6):1031-1037.

9. Wu W, Guo J, An S, Peng G, Ren Y, Xia L, Zhou B: Comparison of Two Hybrid Models for Forecasting the Incidence of Hemorrhagic Fever with Renal Syndrome in Jiangsu Province, China. Plos One 2015, 10(8):e0135492.

10. Bi P, Wu X, Zhang F, Parton KA, Tong S: Seasonal rainfall variability, the incidence of hemorrhagic fever with renal syndrome, and prediction of the disease in low-lying areas of China. American Journal of 
Epidemiology 1998, 148(3):276-281.

11. Olsson GE, Hjertqvist M, Lundkvist A, Hörnfeldt B: Predicting High Risk for Human Hantavirus Infections, Sweden. Emerging Infectious Diseases 2009, 15(1):104-106.

12. Wei Y, Wang Y, Li X, Qin P, Lu Y, Xu J, Chen S, Li M, Yang Z: Meteorological factors and risk of hemorrhagic fever with renal syndrome in Guangzhou, southern China, 2006-2015. PLoS Negl Trop Dis 2018, 12(6):e0006604.

13. Jiang F, Wang L, Wang S, Zhu L, Dong L, Zhang Z, Hao B, Yang F, Liu W, Deng Y et al: Meteorological factors affect the epidemiology of hemorrhagic fever with renal syndrome via altering the breeding and hantavirus-carrying states of rodents and mites: a 9 years' longitudinal study. Emerging microbes \& infections 2017, 6(11):e104.

14. Zhang WY, Guo WD, Fang LQ, Li CP, Peng B, Glass GE, Jiang JF, Sun SH, Quan Q, Wei L: Climate variability and hemorrhagic fever with renal syndrome transmission in Northeastern China. Environ Health Perspect 2010, 118(7):915-920.

15. Liu Q, Liu X, Jiang B, Yang W: Forecasting incidence of hemorrhagic fever with renal syndrome in China using ARIMA model. Bmc Infectious Diseases 2011, 11(1):218.

16. Wang T, Zhou Y, Wang L, Huang Z, Cui F, Zhai S: Using autoregressive integrated moving average model to predict the incidence of hemorrhagic fever with renal syndrome in Zibo, China, 2004-2014. Japanese Journal of Infectious Diseases 2015, 69(4):279-284.

17. Xiao D, Wu K, Tan X, Le J, Li H, Yan Y, Xu Z: Modeling and Predicting Hemorrhagic Fever with Renal Syndrome Trends Based on Meteorological Factors in Hu County, China. Plos One 2015, 10(4):e0123166.

18. Zheng YL, Zhang LP, Zhang XL, Wang K, Zheng YJ: Forecast Model Analysis for the Morbidity of Tuberculosis in Xinjiang, China. Plos One 2015, 10(3):e0116832.

19. Wongkoon S, Jaroensutasinee M, Jaroensutasinee K: Development of temporal modeling for prediction of dengue infection in northeastern Thailand. Asian Pacific Journal of Tropical Medicine 2012, 5(3):249-252.

20. Liu L, Luan RS, Yin F, Zhu XP, Q. LÜ: Predicting the incidence of hand, foot and mouth disease in Sichuan province, China using the ARIMA model. Epidemiology \& Infection 2016, 144(1):144-151.

21. Zhang WY, Fang LQ, Jiang JF, Hui FM, Glass GE, Lei Y, Xu YF, Zhao WJ, Hong Y, Wei L: Predicting the risk of hantavirus infection in Beijing, People's Republic of China. American Journal of Tropical Medicine \& Hygiene 2009, 80(4):678-683.

22. Xiang J, Hansen A, Liu Q, Tong MX, Liu X, Sun Y, Cameron S, Hanson-Easey S, Han GS, Williams C et al: Impact of meteorological factors on hemorrhagic fever with renal syndrome in 19 cities in China, 2005- 
2014. The Science of the total environment 2018, 636:1249-1256.

23. Cobb GW: On a measure of lack of fit in time series models. Biometrika 1978, 65(2):297-303.

24. Fang LQ, Wang XJ, Liang S, Li YL, Song SX, Zhang WY, Qian Q, Li YP, Wei L, Wang ZQ: Spatiotemporal Trends and Climatic Factors of Hemorrhagic Fever with Renal Syndrome Epidemic in Shandong Province, China. Plos Neglected Tropical Diseases 2010, 4(8):e789.

25. Bai Y, Xu Z, Lu B, Sun Q, Tang W, Liu X, Yang W, Xu X, Liu Q: Effects of Climate and Rodent Factors on Hemorrhagic Fever with Renal Syndrome in Chongqing, China, 1997-2008. Plos One 2015, 10(7):e0133218.

26. Guan P, Huang D, He M, Shen T, Guo J, Zhou B: Investigating the effects of climatic variables and reservoir on the incidence of hemorrhagic fever with renal syndrome in Huludao City, China: a 17-year data analysis based on structure equation model. Bmc Infectious Diseases 2009, 9(1):109.

27. Bi P, Tong S, Donald K, Parton K, Ni J: Climatic, reservoir and occupational variables and the transmission of haemorrhagic fever with renal syndrome in China. International Journal of Epidemiology 2002, 31(1):189.

28. Liu X, Jiang B, Gu W, Liu Q: Temporal trend and climate factors of hemorrhagic fever with renal syndrome epidemic in Shenyang City, China. Bmc Infectious Diseases 2011, 11(1):331.

29. Engelthaler DM, Mosley DG, Cheek JE, Levy CE, Komatsu KK, Ettestad P, Davis T, Tanda DT, Miller L, Frampton JW: Climatic and environmental patterns associated with hantavirus pulmonary syndrome, Four Corners region, United States. Emerging Infectious Diseases 1999, 5(1):87-94.

30. Tamerius JD, Wise EK, Uejio CK, Mccoy AL, Comrie AC: Climate and human health: synthesizing environmental complexity and uncertainty. Stochastic Environmental Research \& Risk Assessment 2007, 21(5):601-613.

31. Hjelle B, Glass GE: Outbreak of hantavirus infection in the Four Corners region of the United States in the wake of the 1997-1998 El Nino-southern oscillation. Journal of Infectious Diseases 2000, 181(5):1569-1573.

32. Tian HY, Yu PB, Luis AD, Bi P, Cazelles B, Laine M, Huang SQ, Ma CF, Zhou S, Wei J et al: Changes in rodent abundance and weather conditions potentially drive hemorrhagic fever with renal syndrome outbreaks in Xi'an, China, 2005-2012. PLoS Neg/ Trop Dis 2015, 9(3):e0003530.

\section{Tables}

Table 1 Comparisons of plausible ARIMA models 


\begin{tabular}{|c|c|c|c|c|c|c|c|}
\hline \multirow[t]{2}{*}{ ARIMA model } & \multicolumn{5}{|l|}{$\begin{array}{l}\text { Model fit } \\
\text { statistics }\end{array}$} & \multirow{2}{*}{$\begin{array}{l}\text { Ljung- } \\
\text { Box } \\
\text { Q(18) } \\
\text { Statistics }\end{array}$} & \multirow[b]{2}{*}{ Sig } \\
\hline & R-squared & RMSE & MAE & MaxAE & $\begin{array}{l}\text { Normalized } \\
\text { BIC }\end{array}$ & & \\
\hline $\begin{array}{l}\text { ARIMA ( 0ख1ه0)( } \\
0 \rrbracket 1 \rrbracket 0) 12\end{array}$ & -0.178 & 3.944 & 2.857 & 16.077 & 2.777 & 102.159 & 0.000 \\
\hline $\begin{array}{l}\operatorname{ARIMA}(0 \rrbracket 1 \rrbracket 1)( \\
0 \rrbracket 1 \rrbracket 1) 12\end{array}$ & 0.264 & 3.138 & 2.338 & 11.558 & 2.385 & 18.173 & 0.314 \\
\hline $\begin{array}{l}\operatorname{ARIMA}(0 \rrbracket 1 \rrbracket 2)( \\
0 \rrbracket 1 \rrbracket 2) 12\end{array}$ & 0.294 & 3.094 & 2.264 & 10.076 & 2.422 & 17.787 & 0.217 \\
\hline $\begin{array}{l}\text { ARIMA }(1 \otimes 1 \otimes 0)( \\
1 \otimes 1 \otimes 0) 12\end{array}$ & 0.131 & 3.409 & 2.560 & 12.643 & 2.550 & 41.689 & 0.000 \\
\hline 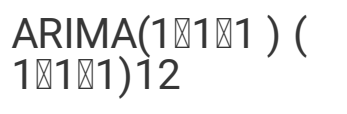 & 0.289 & 3.105 & 2.274 & 10.094 & 2.429 & 18.660 & 0.178 \\
\hline $\begin{array}{l}\operatorname{ARIMA}(1 \rrbracket 1 \rrbracket 2)( \\
1 \rrbracket 1 \otimes 2) 12\end{array}$ & 0.303 & 3.095 & 2.264 & 10.027 & 2.488 & 16.775 & 0.158 \\
\hline $\begin{array}{l}\operatorname{ARIMA}(2 \rrbracket 1 \otimes 0)( \\
2 \rrbracket 1 \rrbracket 0) 12\end{array}$ & 0.232 & 3.227 & 2.452 & 13.343 & 2.506 & 30.340 & 0.007 \\
\hline $\begin{array}{l}\operatorname{ARIMA}(2 \otimes 1 \otimes 1)( \\
2 \bigotimes 1 \otimes 1) 12\end{array}$ & 0.291 & 3.120 & 2.263 & 9.833 & 2.504 & 18.007 & 0.115 \\
\hline
\end{tabular}

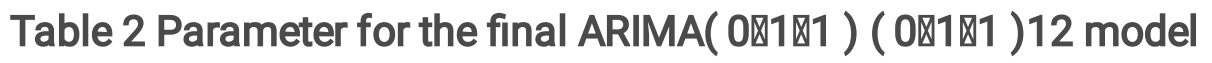

\begin{tabular}{lllll} 
Parameter & Estimate & SE & t & Sig. \\
\hline MA & 0.730 & 0.061 & 12.041 & 0.000 \\
MA, Seasonal & 0.569 & 0.080 & 7.141 & 0.000
\end{tabular}

Table 3 Comparison of predicted HFRS cases and actual values for Anqiu, during January-December 2014 
Month

\section{Cases}

Actual value Predicted value 95\% Confidence Interval Noise Residuals

\begin{tabular}{|c|c|c|c|c|}
\hline January & 3.00 & 3.67 & 邓-2.13-9.47】 & -0.67 \\
\hline February & 4.00 & 2.16 & 邓-3.64-7.95凹 & 1.84 \\
\hline March & 1.00 & 0.95 & 囚-4.85-6.74凹 & 0.05 \\
\hline April & 3.00 & 2.88 & $\rrbracket-4.92-6.68 \rrbracket$ & 0.12 \\
\hline May & 1.00 & 2.42 & ه-3.38-8.21》 & -1.42 \\
\hline June & 2.00 & 3.95 & 囚-1.85-9.74凹 & -1.95 \\
\hline July & 1.00 & 0.71 & ه-5.08-6.51】 & 0.29 \\
\hline August & 3.00 & 0.60 & ه-5.20-6.39ه & 2.40 \\
\hline September & 0.00 & 3.10 & ه-2.70-8.89ه & -3.10 \\
\hline October & 4.00 & 8.17 & $\unrhd 2.38-13.97 \rrbracket$ & -4.17 \\
\hline November & 6.00 & 7.03 & $\nabla 1.24-12.83 \rrbracket$ & -1.03 \\
\hline December & 3.00 & 1.86 & \-3.94-7.65】 & 1.14 \\
\hline
\end{tabular}

Table 4 Multiple linear analysis of meteorological factors and monthly HFRS incidence

$\begin{array}{lll}\text { Variable } & \text { Unstandardized } & \text { Standardized } \\ \text { Coefficients } & \text { Coefficients }\end{array}$

$95.0 \%$ Confidence Interval for B

B Beta Beta $\quad t \quad$ Sig. $\begin{aligned} & \text { Lower } \\ & \text { Bound }\end{aligned} \begin{aligned} & \text { Upper } \\ & \text { Bound }\end{aligned}$

\begin{tabular}{llllllll} 
Temperature & 0.001 & 0.004 & 0.024 & 0.269 & 0.788 & -0.007 & 0.009 \\
\hline Precipitation & -0.002 & 0.001 & -0.241 & -2.603 & 0.010 & -0.003 & 0.000 \\
\hline Wind speed & 0.189 & 0.052 & 0.262 & 3.631 & 0.000 & 0.086 & 0.291 \\
\hline Constant & 0.055 & 0.138 & - & 0.399 & 0.691 & -0.218 & 0.328
\end{tabular}

\section{Figures}




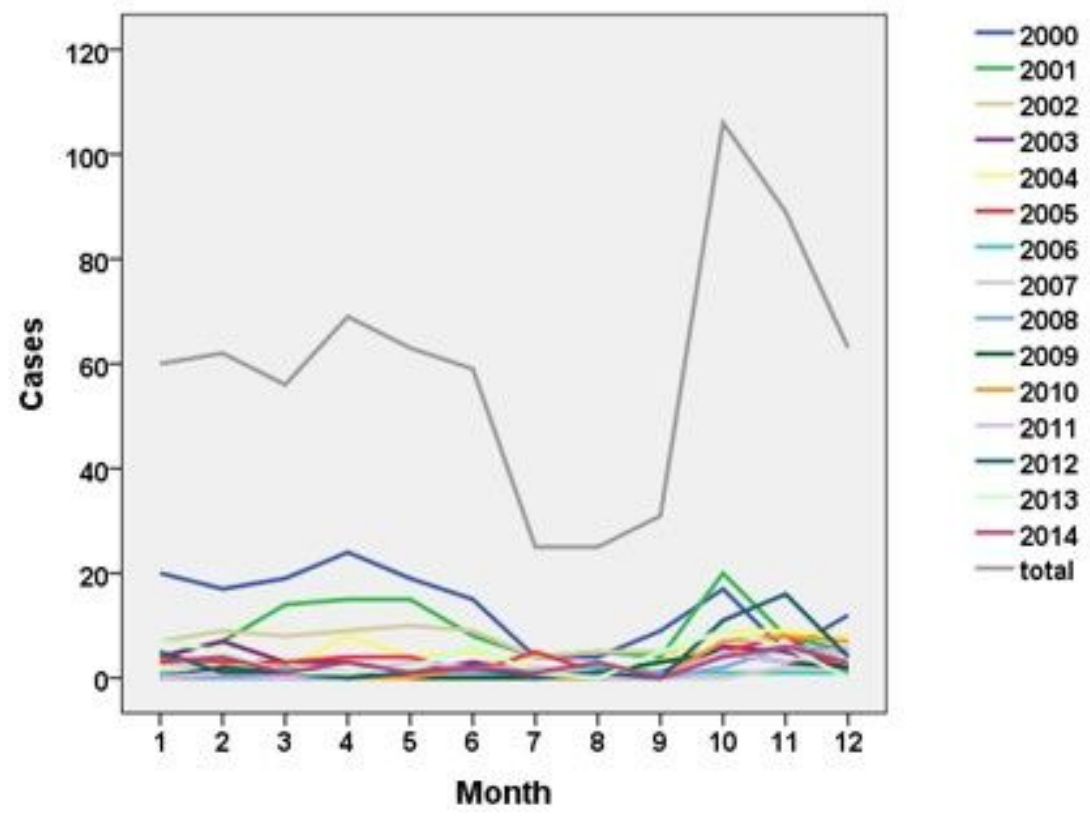

\section{Figure 1}

Monthly distribution of HFRS cases in Anqiu City, 2000-2014

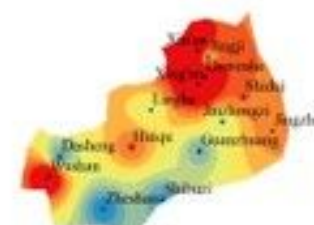

(a) 2000

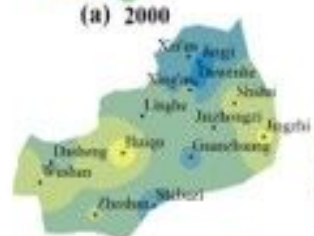

(e) 2004

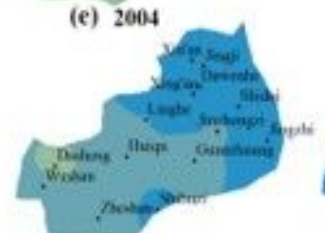

(i) 2008

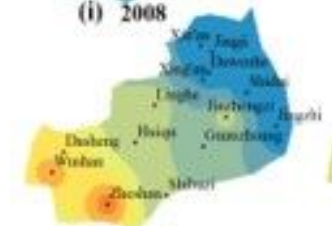

(m) 2012

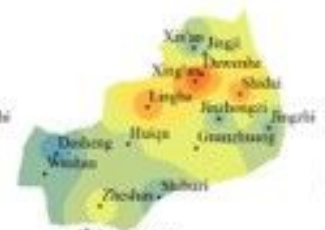

(b) 2001

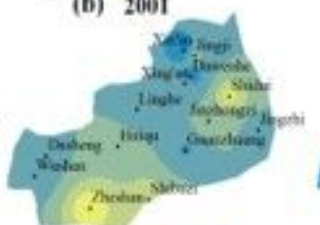

(f) 2005

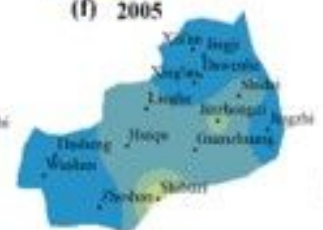

(j) 2009

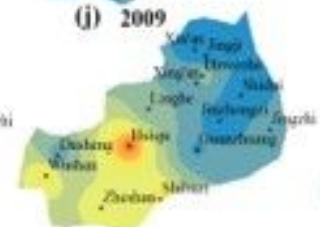

(a) 2013

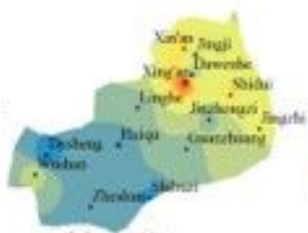

(c) 2002

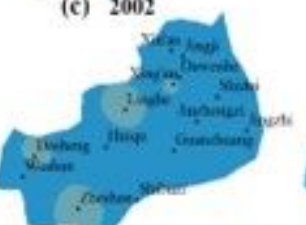

(g) 2006

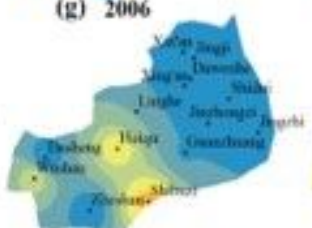

(k) 2010

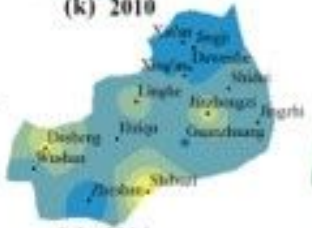

(o) 2014

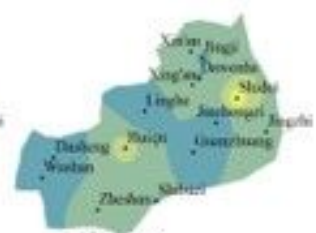

(d) 2003

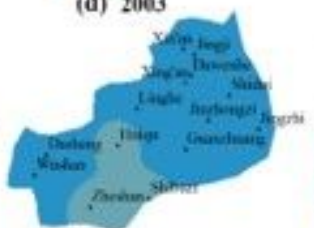

(h) 2007

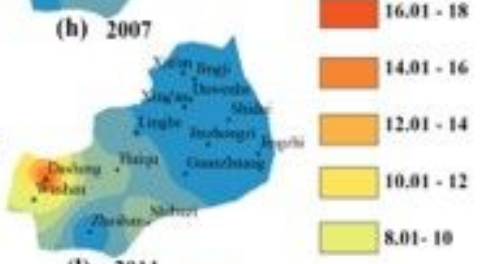

(1) 2011

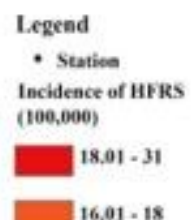

Incidence of HFRS

$18.01-31$

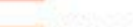

\begin{tabular}{|l}
\hline $6.01-8$ \\
\hline $4.01-6$ \\
$2.01-4$ \\
$0 \cdot 2$
\end{tabular}

(p) 2000-2014

\section{Figure 2}

The spatial distribution of HFRS incidence in different year and their annualized average incidence in each town, 2000-2014 


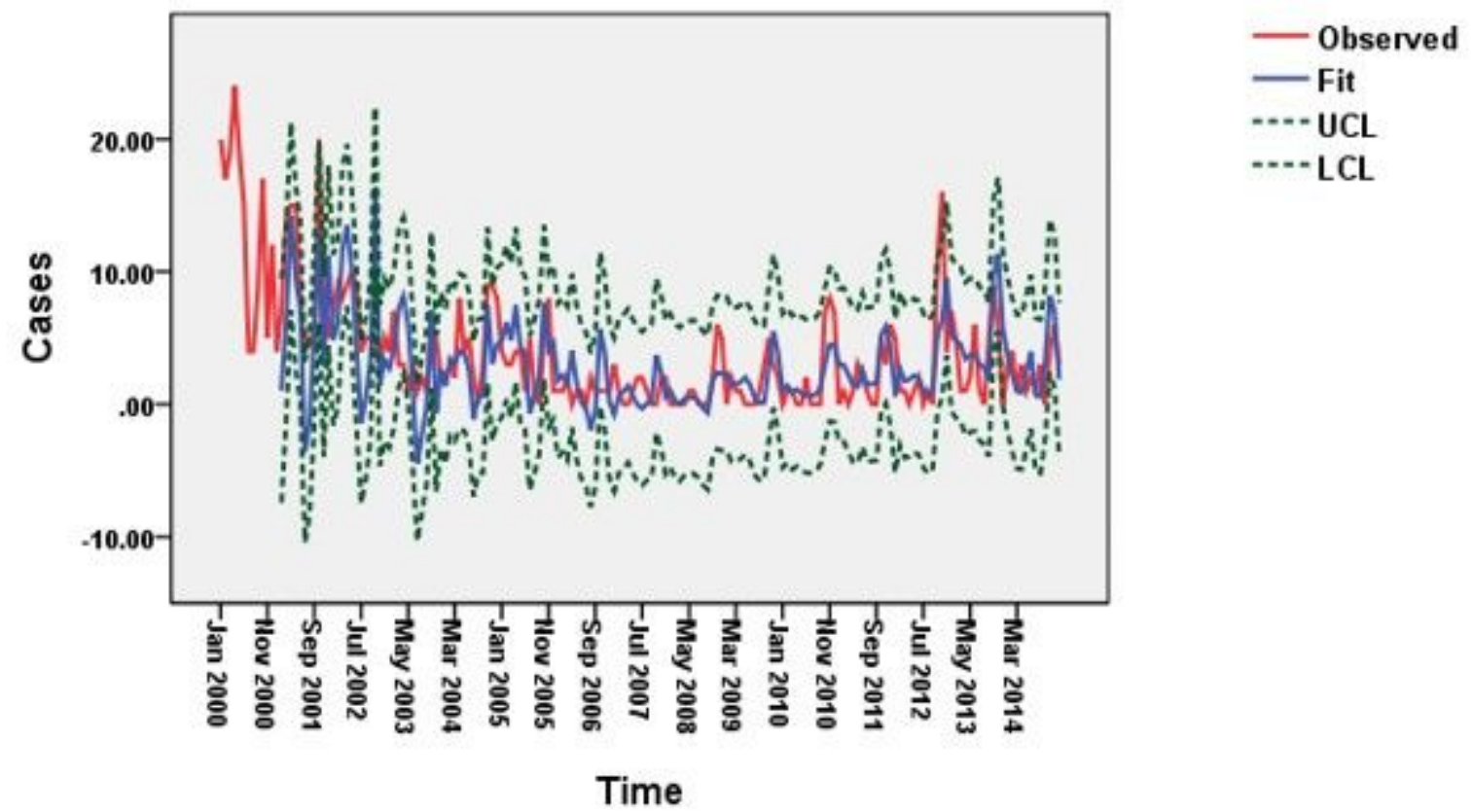

Figure 3

Fitted, predicted and actual cases of HFRS in Anqiu City from 2000 to 2014.Red solid line: observed values; blue solid line: $\operatorname{ARIMA}(0,1,1) \times(0,1,1) 12$ model fitted curve; green dashed lines: $95 \%$ confidence intervals of fitted values. UCL, upper confidence limits; LCL, lower confidence limits. 